\title{
Análise do conhecimento masculino frente ao câncer de mama
}

\author{
Analysis of male knowledge against breast cancer \\ Análisis del conocimiento masculino frente al câncer de mama
}

Recebido: 22/06/2021 | Revisado: 30/06/2021 | Aceito: 01/07/2021 | Publicado: 14/07/2021

Diana da Silva Telésforo
ORCID: https://orcid.org/0000-0002-5120-640X
Centro Universitário de Viçosa, Brasil
E-mail: dianasilvat135@gmail.com
Marli do Carmo Cupertio
ORCID: https://orcid.org/0000-0001-5790-0622
Faculdade Dinâmica do Vale do Piraga, Brasil
E-mail: marli.cupertio@ufv.br
Ramon Repolês Soares
ORCID: https://orcid.org/0000-0001-6200-5606
Centro Universitário de Viçosa, Brasil
E-mail: ramonrepoles@ hotmail.com
Emília Pio da Silva
ORCID: https://orcid.org/0000-0001-8130-5196
Centro Universitário de Viçosa, Brasil
E-mail: emiliapio@ univicosa.com.br

\begin{abstract}
Resumo
O câncer de mama é uma doença com taxa de incidência progressiva com o avançar da idade, podendo acometer tanto mulheres, quanto homens. A falta de conhecimento sobre o câncer de mama em homens impede que a população masculina busque por serviços de saúde, desencadeando um diagnóstico tardio, reduzindo as chances de cura. Diante disso, o objetivou- se analisar a percepção dos homens à cerca da ocorrência do câncer de mama no sexo masculino, descrevendo o perfil epidemiológico, através das características sociais e demográficas dos participantes da pesquisa. Para isso, foi desenvolvido um questionário estruturado com questões relativas aos dados sociodemográficos e questões sobre aspectos específicos do câncer de mama. Os dados coletados foram analisados por meio de estatística descritiva com o auxílio do programa Microsoft Office Excel. Como resultado, foi observado que a maioria dos homens conhecem a doença, contudo, desconhecem a ocorrência no público masculino. O desconhecimento dos participantes pode estar relacionado com o fato da maioria das campanhas de prevenção serem dirigidas ao público feminino e pela falta de conhecimento masculino ao se considerarem invulneráveis, e procuram o médico somete na presença de sintomas da doença. Conclui-se que a falta de informação e das campanhas de prevenção para a doença direcionadas ao público masculino geram uma grande problemática, contribuindo para o descuido na busca por cuidados de saúde preventivos.
\end{abstract}

Palavras-chave: Câncer de mama; Gênero masculino; Prevenção à saúde.

\begin{abstract}
Breast cancer is a disease with a progressive incidence rate with advancing age, which can affect both women and men. The lack of knowledge about breast cancer in men prevents the male population from seeking health services, triggering a late diagnosis, reducing the chances of cure. Therefore, the objective was to analyze the perception of men about the occurrence of breast cancer in men, describing the epidemiological profile, through the social and demographic characteristics of the research participants. For this, a structured questionnaire was developed with questions related to sociodemographic data and questions about specific aspects of breast cancer. The collected data were analyzed using descriptive statistics with the aid of the Microsoft Office Excel program. As a result, it was observed that most men know the disease, however, they are unaware of the occurrence in the male audience. The participants' lack of knowledge may be related to the fact that most prevention campaigns are aimed at the female public and the lack of male knowledge when considering themselves invulnerable, and they seek the doctor only in the presence of symptoms of the disease. It is concluded that the lack of information and prevention campaigns for the disease directed at the male public generate a great problem, contributing to the carelessness in the search for preventive health care.
\end{abstract}

Keywords: Breast cancer; Male gender; Health prevention.

\section{Resumen}

El câncer de mama es uma enfermedad com uma tasa de incidencia progressiva con la edad, que puede afectar tanto a mujeres como a hombres. La falta de conocimiento sobre el cáncer de mama en los hombres impide que una 
población masculina busque servicios de salud, lo que desencadena un diagnóstico tardio, como las possibilidades de curación. Por tanto, el objetivo fue analizar la percepción de los hombres sobre la ocurrencia de cáncer de mama en hombres, describiendo el perfil epidemiológico, a través de las características sociodemográficas de los participantes de la investigación. Para ello, se desarrolló um cuestionario estructurado com preguntas relacionadas com datos sociodemográficos y preguntas sobre aspectos específicos del cáncer de mama. Los datos recolectados se formaron utilizando estadística descriptiva con la ayuda del programa Microsoft Office Excel. Como resultado, se observo que la mayoría de los hombres conocen la enfermedad, sin embargo, desconocen la ocurrencia em la audiência masculina. El desconocimiento de los participantes puede estar relacionado com el hecho de que la mayoría de las campañas de prevención están dirigidas al público femenino y al desconocimiento de los hombres al considerarse invulnerables, y solo buscan al médico ante la presencia de sintomas de la enfermedad. Se concluye que la falta de campañas de información y prevención de la enfermedad dirigidas al público masculino genera un gran problema, contribuyendo al descuido em la búsqueda de cuidados de salud preventivos.

Palabras clave: Cáncer de mama; Género masculino; Prevención de la salud.

\section{Introdução}

O câncer de mama é um assunto muito debatido entre as mulheres. Porém, os homens, assim como as mulheres, possuem glândulas mamárias e hormônios femininos, ainda que em menor quantidade. Com isso, apesar de raro, o câncer de mama também pode acometê-los. Enquanto vários hormônios nos corpos de meninas e mulheres estimulam o desenvolvimento das glândulas mamárias, nos meninos e homens, normalmente não há esse estímulo, resultando em um tecido mamário rudimentar. Os homens podem desenvolver as glândulas mamárias quando tomam certos medicamentos ou têm níveis anormais de hormônios (Inca, 2019).

De modo geral, as políticas de saúde prestam adequada informação e assistência à mulher na prevenção e no tratamento desta doença. A maioria das campanhas de prevenção e alerta sobre o câncer de mama são direcionadas à população feminina, contudo, os homens também podem ser vítimas das neoplasias mamárias (Ramos et al., 2017). A prevalência da doença aumenta com o avançar da idade e a suspeita clínica pelos profissionais de atenção primária é baixa. Os principais fatores de risco são associados ao histórico familiar em parentes de primeiro grau, suscetibilidade genética, exposição ambiental a agentes químicos e petroquímicos, desequilíbrio hormonal, exposição à radiação ionizante, dentre outras. A suspeita clínica da doença surge por meio da anamnese e pelo exame físico (Azevedo \& Monteiro, 2018).

O câncer mamário é a primeira causa de morte entre as mulheres. Entretanto, a neoplasia de mama masculina é rara, e estima-se que para 100 novos casos de câncer de mama em mulheres, apenas um caso em homens é diagnosticado, o que representa $0,8 \%$ a $1 \%$ de casos de neoplasia mamária. No entanto a taxa de incidência tem crescido nos últimos anos, e nos Estados Unidos são relatados 1500 novos casos, enquanto na década de 70 eram relatados 700 novos casos de câncer masculino por ano (Araújo et al., 2018). O Instituto Nacional do Câncer (INCA) estima que para cada ano do próximo triênio (2020/2022), serão diagnosticados 66.280 novos casos de câncer de mama, sendo cerca de 17.572 em mulheres e 189 em homens (Inca, 2020).

Frequentemente a população masculina tende a protelar a procura por serviços de saúde. Muitas vezes por questões culturais implantadas pela própria sociedade e pela história de transformação na saúde, as atenções são direcionadas à população feminina, por serem progenitoras e desempenharem o papel fundamental na geração de novas vidas. Juntamente a esses fatores, o machismo e o desconhecimento do assunto fizeram com que a população masculina se mantivesse afastada dos serviços de saúde (Ramos et al., 2017).

Outro fator importante é a baixa incidência da patologia. Tal fato pode contribuir para o desconhecimento da doença por parte da população masculina, necessitando de atenção por parte dos serviços de atenção primária na promoção de campanhas de conscientização e esclarecimentos sobre fatores de risco, bem como os sinais e os sintomas iniciais associados a essa patologia, possibilitando assim diagnóstico e terapêutica precoces (Trajano et al., 2018).

O conhecimento dos homens sobre a existência da doença e as informações sobre as manifestações clínicas pode 
prevenir a ocorrência do câncer de mama. O esclarecimento da população sobre a doença ocasionaria a procura mais rápida por atendimento médico, auxiliando no diagnóstico precoce e, consequentemente, aumentando as chances de sobrevida, com melhor prognóstico da doença (Salomon, 2015). Assim, objetivou-se analisar o conhecimento da população masculina à cerca da ocorrência do câncer de mama no sexo masculino, buscando-se descrever as características sociais demográficas dos participantes da pesquisa.

\section{Metodologia}

Tratou-se de uma pesquisa aplicada, de natureza quantitativa, quantos aos objetivos tem-se uma pesquisa exploratória e descritiva. De acordo com Köche (2011) a pesquisa exploratória não visa a relação entre variáveis, mas o levantamento da presença das variáveis juntamente com sua caracterização quantitativa, além disso, na pesquisa descritiva não há a manipulação das variáveis estudadas.

A pesquisa foi realizada na microrregião de Viçosa - MG. Segundo o Consórcio Intermunicipal de Saúde da Microrregião de Viçosa (CIS-MIV), a microrregião é composta por 10 (dez) municípios, sendo eles: Araponga, Canaã, Cajuri, Coimbra, Paula Cândido, Pedra do Anta, Porto Firme, São Miguel do Anta, Teixeiras e Viçosa. A missão do CIS-MIV é buscar a integração entre os municípios consorciados, planejando, adotando e executando com maior eficiência as ações e serviços necessários à população assistida pelos serviços complementares de assistência à saúde, conforme preceitua o Sistema Único de Saúde - SUS (CIS-MIV, 2020).

A pesquisa envolveu homens selecionados de forma aleatória, respeitando os seguintes critérios de inclusão: possuir idade igual ou superior a 18 anos e aceitar participar da pesquisa. Para a coleta de dados utilizou- se um questionário estruturado e desenvolvido especificamente para a pesquisa em questão, elaborado com base no estudo de Souza (2015). A entrevista foi disponibilizada no Google Forms. O questionário foi enviado via rede social (Facebook e WhatsApp) aos participantes para ser respondido de forma online, sendo disponibilizado por um período de 30 dias. O período da coleta de dados foi de 04/03/2021 a 04/04/2021.

O instrumento de pesquisa dividiu-se em duas partes. A primeira com questões relativas aos dados sociodemográficos, com o objetivo de caracterizar os sujeitos do estudo. Já a segunda parte abordou aspectos específicos ao câncer de mama. As questões utilizadas no questionário foram de respostas únicas, nas quais apenas um tipo de resposta poderia ser escolhido; e questões dicotômicas, em que possuiu apenas duas opções de resposta (sim/não).

Os dados foram analisados utilizando estatística descritiva com auxílio do programa Microsoft Office Excel, em seguida esses dados foram apresentados em tabelas.

O projeto de pesquisa foi enviado para análise do Comitê de Ética em Pesquisa (CEP), segundo os procedimentos da Plataforma Brasil, avaliado e aprovado pelo CEP do Centro Universitário de Viçosa- FACISA/UNIVICOSA, de acordo com o Parecer 4.573.946.

\section{Resultados e Discussão}

A pesquisa incluiu 124 homens, residentes nos municípios da microrregião de Viçosa, entre as faixas etárias de 18 a 70 anos. Na amostra, a predominância foi de homens residentes do município de Viçosa (68,5\%), solteiros (75\%) e com ensino médio completo $(31,5 \%)$ e superior incompleto respectivamente (Tabela 1$)$. 
Tabela 1: Distribuição amostral quanto aos dados sociodemográficos dos participantes da pesquisa $(\mathrm{n}=124)$.

\begin{tabular}{|c|c|c|c|}
\hline Características & Variável & $\mathbf{n}$ & $\%$ \\
\hline \multirow[t]{4}{*}{ Idade } & $18-31$ & 86 & 69,4 \\
\hline & $32-45$ & 23 & 18,5 \\
\hline & $46-58$ & 13 & 10,5 \\
\hline & $59-70$ & 2 & 1,6 \\
\hline \multirow[t]{3}{*}{ Estado Civil } & Solteiro & 93 & 75 \\
\hline & Casado & 24 & 19,4 \\
\hline & Outros & 7 & 5,6 \\
\hline \multirow[t]{9}{*}{ Cidade } & Araponga & 6 & 4,8 \\
\hline & Canaã & 6 & 4,8 \\
\hline & Cajuri & 1 & 0,8 \\
\hline & Coimbra & 1 & 0,8 \\
\hline & Paula Candido & 3 & 2,4 \\
\hline & Porto Firme & 9 & 7,3 \\
\hline & São M. do Anta & 6 & 4,8 \\
\hline & Teixeiras & 7 & 5,6 \\
\hline & Viçosa & 85 & 68,5 \\
\hline \multirow[t]{8}{*}{ Escolaridade } & Não alfabetizado & 1 & 0,8 \\
\hline & Ensino fundamental incompleto & 4 & 3,2 \\
\hline & Ensino fundamental completo & 5 & 4,0 \\
\hline & Ensino médio incompleto & 5 & 4,0 \\
\hline & Ensino médio completo & 41 & 33,1 \\
\hline & Ensino superior incompleto & 41 & 33,1 \\
\hline & Ensino superior completo & 12 & 9,7 \\
\hline & Pós - graduação & 15 & 12,1 \\
\hline TOTAL & & 124 & 100 \\
\hline
\end{tabular}

Fonte: Dados da pesquisa (2021).

Por meio da Tabela 1, verificou-se que a maioria dos participantes eram adultos jovens (87,9\%), apesar desta faixa etária não representar um grupo de risco, seria um público, favorável para orientação quanto à necessidade de adoção de medidas preventivas. Segundo Araújo et al. (2018), a prevalência da doença aumenta conforme a idade, sendo rara antes dos 30 anos. A média de diagnóstico dos pacientes gira em torno de 60 a 70 anos, que é considerado um diagnóstico tardio quando comparado às mulheres, reduzindo, assim, as chances de cura da doença, por isso, a conscientização dos homens acerca da doença é imprescindível.

É sabido que a maioria dos homens não procuram por serviços preventivos de saúde, pois normalmente buscam ajuda médica apenas para alívio de sintomas já instalados. Para Trajano et al. (2018), homens casados possuem maior estímulo na busca pelo serviço de saúde, uma vez que as mulheres se preocupam mais com a prevenção das doenças, estimulando seus maridos a realizarem consultas preventivas.

Os resultados desta pesquisa revelaram que os homens não relacionavam o câncer de mama ao gênero masculino. A maioria $(85,5 \%)$ não conheciam a causa da doença (Tabela 2). A falta de conhecimento por parte da população masculina sobre o acometimento do câncer de mama traz um viés negativo para a prevenção e detecção precoce da doença, sendo assim os homens acabam recebendo o diagnóstico tardiamente, quando a doença já está em estágios avançados (Ramos et al., 2017). 
Tabela 2: Conhecimento dos homens analisados quantos aspectos relacionados ao câncer de mama.

\begin{tabular}{|c|c|c|c|}
\hline Característica & Variável & $\mathbf{N}$ & $\%$ \\
\hline \multirow[t]{2}{*}{ Costuma buscar o serviço de saúde com frequência? } & Sim & 33 & 26,6 \\
\hline & Não & 91 & 73,4 \\
\hline \multirow[t]{2}{*}{ Você sabe o significado de câncer de mama? } & Sim & 89 & 71,8 \\
\hline & Não & 35 & 28,2 \\
\hline Você tem conhecimento sobre os tipos de câncer & Sim & 70 & 56,5 \\
\hline existentes? & Não & 54 & 43,5 \\
\hline Você tem conhecimento a respeito de câncer de mama & Sim & 32 & 25,8 \\
\hline masculino? & Não & 92 & 74,2 \\
\hline Você tem conhecimento a respeito dos fatores de risco & Sim & 35 & 28,2 \\
\hline para a ocorrência do câncer de mama? & Não & 71 & 71,8 \\
\hline
\end{tabular}

\begin{tabular}{cccc}
\hline $\begin{array}{c}\text { Você tem conhecimento sobre o tratamento do câncer de } \\
\text { mama masculino? }\end{array}$ & Sim & 17 & 13,7 \\
& & 107 & 86,3 \\
\hline $\begin{array}{c}\text { Já recebeu alguma orientação a respeito desta patologia } \\
\text { pelos profissionais da saúde da sua UBS? }\end{array}$ & Sim & 10 & 8,1 \\
\hline
\end{tabular}

\begin{tabular}{cccc}
\hline Você sabe o que causa o câncer de mama? & Sim & 18 & 14,5 \\
& Não & 106 & 85,5 \\
\hline Não realiza o auto exame das mamas por qual motivo? & Não sei da & 76 & 61,3 \\
necessidade do & exame/ & 38,7 \\
& Não sei fazer o & 48 & exame
\end{tabular}

\begin{tabular}{lcc}
\hline TOTAL & 124 & 100 \\
\hline
\end{tabular}

Fonte: Dados da pesquisa (2021).

Quando questionados se procuravam o serviço de saúde com frequência, a maioria respondeu que não $(73,4 \%)$ e apenas 26,6\% afirmaram que sim. Segundo Ramos et al. (2017), a não adesão à saúde preventiva torna os homens muito mais suscetíveis a morbidades e agravos.

De acordo com o Ministério da Saúde, considerando a exposição a um maior nível vulnerabilidade e a elevadas taxas de morbimortalidade, fica cada vez mais evidente que os homens não possuem o costume de buscar por serviços de atenção primária à saúde. Se, os homens tivessem o hábito de realizar consultas preventivas, com certeza haveria uma redução dos agravos na sua saúde (Ministério da Saúde, 2008).

Conforme abordado por Carvalho et al. (2013), essa falta de procura pelo serviço de saúde tem fundamentos em uma cultura machista. Silva et al. (2012) afirma que este preconceito é uma construção social devido à vinculação do homem à 
imagem de ausência de cuidado com a saúde. Conforme explicado pelo Ministério da Saúde, as doenças tendem a ser consideradas um sinal de fragilidade para os homens, visto que eles não as reconhecem como inerentes à sua própria condição biológica, o que contribui para a menor prestação de cuidados a eles mesmos (Ministério da Saúde, 2008).

Ao serem questionados se sabiam o significado do câncer de mama, 71,8\% afirmaram que sim e 28,2\% não sabiam o que significava essa patologia. Ao serem perguntados se conheciam os tipos de câncer existentes 56,5\% afirmaram que sim. Apesar da maioria dos homens conhecerem o significado do câncer de mama, muitos não sabem da possibilidade de ocorrência no público masculino. O não reconhecimento pelos homens dessa possibilidade em desenvolver o câncer de mama acaba retardando a procura por diagnóstico precoce e de continuidade no tratamento para uma possível cura para a doença. Essa resistência tende a se tornar maior quando o homem é idoso, justamente a faixa etária em que prevalece esse tipo de câncer mamário (Cypriano, 2017).

O câncer de mama geralmente se manifesta por meio do surgimento de uma massa indolor e firme no órgão. A retração ou inversão mamilar, o espessamento da pele e a formação de crostas e ulceração são sinais apresentados por cerca de um terço dos pacientes portadores da enfermidade; enquanto a secreção mamilar pode ocorrer em cerca de $14 \%$ dos acometidos (Leme \& Souza, 2006). Os homens geralmente não observam as alterações que ocorrem em seu corpo, o que acaba por prorrogar o diagnóstico da doença.

Apesar de 71,8\% dos participantes afirmaram que conhece o significado da doença, a maioria $(74,2 \%)$ desconhecia à ocorrência do câncer de mama no gênero masculino. Esse dado nos permite afirmar que os homens não têm acesso à informação ou não procuram se informar quanto ao câncer de mama, talvez pela crença de que a doença acomete apenas mulheres. Isso pode aumentar a morbimortalidade pela doença entre os homens.

Um dos motivos para que isso aconteça é a falta de hábito em acessar o serviço de atenção primária, já adentrando aos postos médicos à procura de serviços de média e alta complexidade. Essa falta de hábito em procurar o serviço de saúde de maneira rotineira retarda a atenção à saúde e contribui para o agravamento do quadro da doença, limitando a ação apenas ao atendimento, visto que em alguns casos já não é mais possível prevenir e promover a saúde (Cristo \& Cristo, 2019).

Conforme explicado por Dantas et al. (2015), o fato dessa neoplasia ser rara na população masculina e estes se manterem afastados dos serviços de saúde, permitem que a doença se torne mais agressiva. Em maioria, o diagnóstico é feito em idades e estados mais avançados, quando comparados às mulheres, sendo, portanto, necessária uma detecção mais precoce.

De acordo com Figueiredo (2005), estudos apontam que as próprias instituições de saúde são reprodutoras do imaginário social de gênero. Isso porque os serviços de saúde oferecem menor tempo de seus profissionais aos homens quando em comparação com as mulheres. Percebe-se, portanto, que o espaço é voltado mais para o público feminino, segmento que, por anos foi considerado prioridade absoluta. Os cartazes fixados nas unidades de saúde, por exemplo, expressam campanhas de aleitamento materno, pré-natal, exames preventivos do colo de útero e da mama, dentre outros. Essas campanhas são extremamente importantes para as mulheres e para as crianças, mas campanhas como a prevenção do câncer de mama (o "Outubro Rosa") deveriam ser extensas ao público masculino, para que eles se sentissem pertencidos a esse segmento e se sentissem incentivados à procurar atendimento médico precocemente.

A falta de conhecimento dos homens quanto ao câncer de mama fica evidente no fato de $71,8 \%$ relatarem não conhecer os fatores de risco para a doença e $86,3 \%$ desconhecerem as modalidades de tratamento. Neste mesmo cenário, outra questão ajuda explicar a falta de conhecimento masculina, pois quase $100 \%$ dos entrevistados $(91,9 \%)$ disseram não ter recebido dos profissionais de saúde das Unidades Básicas de Saúde (UBSs) orientações sobre a doença. Segundo Oliveira et al. (2019), os profissionais de saúde não reconhecem os homens como protagonistas do cuidado com a própria saúde, não estimulando esse cuidado neles, principalmente, no aspecto da prevenção e promoção à saúde, pois, já se acostumaram com o comportamento masculino de se considerarem invulneráveis. 
Ao serem indagados sobre o que fazer para evitar o câncer de mama, 31,5\% afirmaram que era preciso ter hábitos de vida saudáveis; $27,4 \%$ não sabem o que fazer; $17,7 \%$ responderam ir ao médico; $15,3 \%$ citaram a mamografia, $7,3 \%$ responderam fazer o autoexame da mama rotineiramente. O que se observa é que, apesar de a maioria dos homens acreditar que o câncer de mama é uma doença exclusivamente feminina, eles conhecem as medidas de prevenção, o que é um ponto positivo que facilitaria a conscientização deste público.

De acordo com Vieira et al. (2011), é fundamental que os profissionais de saúde instruam os homens sobre o procedimento de autoexame masculino, que deve ser realizado $1 \mathrm{vez}$ por mês com data definida e mantida, seguindo os mesmos passos orientados às mulheres; uma vez que, por ser simples, rápido, não invasivo e indolor, torna-se bastante eficaz para diminuir as chances de complicações, visto que pode detectar o nódulo precocemente.

Portanto, segundo Nogueira et al. (2014) e Moreira \& Carvalho (2016), o papel do enfermeiro é muito importante no processo educativo, por meio da oferta de informações relevantes à prevenção do câncer. Neste caso, o profissional de saúde representa um ator social fundamental na atenção ao público masculino, possibilitando o desenvolvimento de ações voltadas à promoção da saúde, com o esclarecimento de dúvidas e atuando na contribuição para adesão ao autocuidado.

Quanto ao exame de mamografia, 42,7\% dos participantes informaram que não fizeram o exame porque não foi solicitado pelo médico; $23,4 \%$ disseram não conhecer este exame, $22,6 \%$ não fizeram por não ter conhecimento da necessidade; $10,5 \%$ não fazia por descuido e menos de um por cento $(0,8 \%)$ respondeu que era difícil marcar o exame.

Um dos fatores importantes para que o tratamento do câncer de mama masculino ocorra o mais precocemente possível, evitando assim um prognóstico ruim, é a orientação da população masculina sobre a existência da doença, bem como sobre a importância do autoexame das mamas, o qual colabora com a prevenção e favorece o diagnóstico precoce (Ramos et al., 2017).

\section{Considerações Finais}

A partir dos resultados desta pesquisa, foi possível concluir que a maioria dos homens não relacionava o câncer de mama ao gênero masculino, muitos deles desconheciam a causa da doença. Não relacionar o câncer de mama ao gênero masculino, agrava o quadro da doença dificultando a prevenção, a terapêutica e até mesmo a cura do câncer.

Identificou-se que um dos principais fatores de risco para doença é a baixa adesão dos homens nos serviços preventivos de saúde, visto que, é usual a busca por ajuda médica apenas para alívio de sintomas pré-instalados. É importante considerar ainda, o preconceito enfrentando pelos homens diante do diagnóstico da doença. Além disso, os serviços de atenção primária em saúde têm um papel fundamental nos resultados encontrados, já que existe uma escassez de campanhas preventivas do câncer de mama destinadas ao público masculino.

Desta forma, o papel dos diversos profissionais de saúde, incluindo fisioterapeutas, se faz cada vez mais essencial para a implantação da política de saúde no público masculino, incentivando a prevenção e o cuidado contínuo com a saúde.

Ressalta-se a importância da realização de estudos posteriores com a população masculina que não frequentam as Unidades Básicas de Saúde com o intuito de conhecer o seu perfil epidemiológico, suas manifestações e necessidades em saúde de modo a contribuir para ações e políticas de saúde pública que olhem essa população e suas especificidades.

\section{Referências}

Araújo, I. R. S., Leite, C. B., Amorim, T. O., Silva, A. N. L., Fernandes, R. S. Q. \& Carmo, M. S. (2018). Câncer de mama em homens. Rev. Investig. Bioméd. $10(3), 272-278$.

Azevedo, F. S. \& Monteiro, A. B. P. (2018). Abordagem do câncer de mama masculino na atenção primária: revisão da literatura sobre aspectos epidemiológicos, fatores de risco, manifestações clínicas e encaminhamento precoce ao especialista. Rev. Cient. Esc. Estadual Saúde Pública de Goiás Cândido Santiago, 4 (2), 129-138. 
Carvalho, F. P. B., Silva, S. K. N., Oliveira, L. C., Fernandes, A. C. L., Solano, L. C. \& Barreto, E. L. F. (2013). Conhecimento acerca da Política Nacional de Atenção Integral à Saúde do Homem na estratégia de Saúde da Família. Revista de APS, 16 (4), 386-392.

CISMIV. (2020). Consórcio Intermunicipal de Saúde da Microrregião de Viçosa. http://cismiv.mg.gov.br/

Cypriano, A. S. (2017). Ações promotoras de saúde frente ao câncer de mama masculino: subsídios ao gerenciamento do cuidado de Enfermagem na Atenção Básica. 114f. Dissertação (Mestrado em Enfermagem) - Universidade Federal do Rio de Janeiro, Rio de Janeiro, Brasil.

Cristo, D.A. \& Cristo, S. C. A. Saúde do homem: um estudo realizado na Unidade de Saúde da Pedreira em Belém, Pará. APS em Revista, 1 (2), $162-169$.

Dantas, R. C. O., Pereira, J. B., Alencar, L. D., Sousa, A. K. A. \& Farias, M. C. A. D. (2015). Câncer de mama em homem: uma realidade brasileira. Revista Brasileira de Educação e Saúde, 5 (3), 29-34.

Figueiredo, W. (2005). Assistência à saúde dos homens: um desafio para os serviços de atenção primária. Ciênc. Saúde Coletiva, 10 (1), $105-109$.

Instituto Nacional do Câncer José Alencar Gomes da Silva. (2019). A situação do câncer de mama no Brasil: síntese de dados dos sistemas de informação. https://www.inca.gov.br/publicacoes/livros/situacao-do-cancer-de-mama-no-brasil-sintese-de-dados-dos-sistemas-de-informacao

Instituto Nacional do Câncer José Alencar Gomes da Silva (2020). Tipos de Câncer de Mama. https://www.inca.gov.br/tipos-de-cancer/cancer-de-mama

KÖCHE, J. C. Fundamentos de metodologia científica: teoria da ciência e iniciação científica. Vozes, 2011.

Leme, L. H. S., Souza, G.A. (2006). Câncer de mama em homens: aspectos epidemiológicos, clínicos e terapêuticos. Rev. Ciência Médica, 1 (1), $391-396$.

Ministério da Saúde. (2008). Política Nacional de Atenção Integral à Saúde do Homem. http:/l bvsms.saude.gov.br/bvs/publicacoes/politica_nacional_atencao_homem.pdf

Moreira, M. A. \& Carvalho, C. N. (2016). Atenção Integral à Saúde do Homem: Estratégias utilizadas por Enfermeiras (os) nas Unidades de Saúde da Família do interior da Bahia. Revista Sau. \& Transf. Soc, 7 (3), 121-132.

Nogueira, S. P., Mendonça, J. V. \& Pasqualette, H. A. P. (2014). Câncer de mama em homens. Rev. Bras. Mastologia, 24 (4), 109-114.

Oliveira, D. A. J. (2019). Políticas de Saúde e diagnóstico precoce do câncer de mama no Brasil. Rev. Enferm. Digital Cuidado e Promoção da Saúde, 1 (1), $46-50$.

Ramos, S. S., Rodrigues, L. M. S., Silva, T. A. S. M., Balbino, C. M., Souza, M. M. T. \& Silvino, Z. R. (2017). Conhecimentos, mitos e implicações para o cuidado de enfermagem no câncer de mama masculino. Revista Enfermagem Atual, 83 (1), 67-72.

Salomon, M. F. B., Mendonça, J. V., Pasqualette, H. A. P., Pereira, P. M. S. \& Sondermman, V. R. M. (2015). Câncer de mama no homem. Rev. Bras. Mastologia, 25 (4), 141-145.

Silva, P.A.S., Furtado, M. S., Guilhon, A. B., Souza, N. V. D. O. \& David, H. M. S. L. (2012). A saúde do homem na visão dos enfermeiros de uma unidade básica de saúde. Esc. Anna Nery, 16 (3), 561-568.

Souza, C. N.S. (2015). Rastreamento do Câncer da mama: conhecimento, práticas e resistência em mulheres atendidas na estratégia Saúde da Família. 81f. Dissertação (Mestrado em Saúde e Sociedade) - Universidade do Estado do Rio Grande do Norte, Mossoró, Brasil.

Trajano, J. L. A., Menezes, P. C. M., Caldas, M. L. S., Souza, T. A., Lima, A. B. \& Alves, E. S. R. C. (2018). Avaliação do conhecimento dos homens sobre neoplasia mamária masculino. Temas em Saúde, 1 (1), 373-385.

Vieira, L. C. S., Figueiredo, M. L. F., Sales, R. L. U. B., Lopes, W. M. P. S. \& Avelino, F. V. D. (2015). A política nacional de saúde do homem: uma reflexão sobre a questão de gênero. Revista Enfermagem em Foco, 2 (4), 215-217. 